\title{
Relación del síndrome metabólico con la periodontitis: una revisión sistemática
}

\section{Relation of the metabolic syndrome with the periodontitis: a systematic review}

\author{
María Fernanda Vargas ${ }^{1}$, Shirley Dayana Contreras ${ }^{1}$, Yerly Carolina Rodríguez ${ }^{1}$, \\ Claudia Amaya Bautista', Esther Carolina Angarita'
}

Para citar este artículo: Vargas MF, Contreras SD, Rodríguez YC, Amaya Bautista C, Angarita EC. Relación del síndrome metabólico con la periodontitis: una revisión sistemática. UstaSalud 2016;15:36-42.

Licencia Creative Commons

\section{(c) (i) (\$) $\Theta$} lo tanto, los lectores pueden acceder libremente a los artículos en su formato .pdf, igualmente podrán descargarlos y difundirlos; sin embargo no podrán modificarlos o alterarlos, adicionalmente se debe reconocer la autoría de las personas que figuran en las publicaciones, pero estas no podrán comercializadas.

\begin{abstract}
1 Facultad de Odontología, Universidad Santo Tomás, Bucaramanga

Autor de correspondencia:

María Fernanda Vargas

Correo electrónico:

mafe.var@hotmail.com
\end{abstract}

\section{RESUMEN}

Objetivo: analizar la relación del síndrome metabólico con la periodontitis a través de una revisión sistemática.

Materiales y métodos: se realizó una revisión sistemática soportada en artículos científicos, principalmente de estudios de casos y controles, en cuatro bases de datos, teniendo en cuenta variables que permitieran identificar la relación existente entre el síndrome metabólico y la enfermedad periodontal.

Resultados: en total se encontraron 322 artículos en las bases de datos, de los cuales cinco artículos cumplían con los criterios de inclusión con un grado de coincidencia de $66,6 \%$. De los 5 artículos, dos de ellos estudiaron la influencia del síndrome metabólico sobre la periodontitis, valorando variables como profundidad de surco, sangrado al sondaje, índice gingival, reabsorción ósea, índice de placa, pérdida de inserción - nivel de inserción y los otros tres artículos estudiaron la influencia de la periodontitis sobre el síndrome metabólico, teniendo en cuenta variables como la presencia de ciertos patógenos periodontales, valores de glucosa, índice de masa corporal, presión sanguínea, triglicéridos, lipoproteína de alta y baja densidad (HDL-LDL) y factores inflamatorios.

Conclusión: se establece que el síndrome metabólico (SM) y la periodontitis tienen una relación recíproca, ya que cuando los estudios valoraban la influencia del SM sobre la periodontitis o la influencia de la periodontitis sobre el síndrome metabólico (SM), en ambas situaciones se encontró asociación estrecha entre ellos, al obtener valores de odds ratio (OR) mayores a 1 en las variables influyentes.

Palabras clave: Síndrome metabólico, periodontitis, gingivitis.

\section{ABSTRACT}

Objective: analyze the relationship between Metabolic Syndrome and Periodontitis through a systematic review.

Materials and methods: a systematic review supported on scientific articles mainly of case-control studies was made in four databases taking into account variables such to identify the existing relationship between the metabolic syndrome and periodontal disease.

Results: in total, 322 articles were found in the databases, using the descriptors. Of these, five articles met the inclusion criteria with a degree of coincidence of $66.6 \%$. Of the 5 articles, two of them studied the influence of the metabolic syndrome on periodontitis by assessing variables such as furrow depth, bleeding to the catheter, Gingival index, bone resorption, plaque index, insertion loss-insertion level and other three articles Studied the influence of periodontitis on the metabolic syndrome taking into account variables such as the presence of microorganisms, glucose values, body mass index, blood pressure, triglycerides, high and low density lipoprotein (HDL-LDL), and inflammatory factors.

Conclusion: the study established that the metabolic syndrome and periodontitis have a reciprocal relationship, since when the studies assessed the influence of MS on periodontitis or the influence of periodontitis on MS, in both situations, a close association was found between them when obtaining odd ratios (OR) higher than 1 in the influential variables.

Keywords: Metabolic syndrome, periodontitis, gingivitis. 


\section{INTRODUCCIÓN}

La enfermedad periodontal es una patología inflamatoria con etiología multifactorial que afecta el tejido conectivo y el hueso que soportan los dientes ${ }^{1}$. En Colombia, como resultado del ENSAB IV, la mayor parte de la población $(61,8 \%)$ evidenció periodontitis en sus diferentes grados de severidad, siendo la más frecuente la periodontitis moderada, presente en el $43,5 \%$ de los sujetos, seguida por $10,6 \%$ con periodontitis avanzada, con un $38,2 \%$ de los sujetos que se clasifican como sin periodontitis ${ }^{2}$.

Se ha establecido una relación entre la enfermedad periodontal y las enfermedades sistémicas, en la que se plantea que las enfermedades sistémicas están implicadas en el aumento de la severidad de la enfermedad periodontal o viceversa, entre estas enfermedades sistémicas asociadas a la enfermedad periodontal se ha observado un riesgo aumentado de aparición o progresión de enfermedades cardiovasculares, diabetes, hipertensión, artritis reumatoide, obesidad y algunas enfermedades respiratorias ${ }^{3}$.

La enfermedad periodontal está caracterizada por una respuesta inflamatoria de los tejidos gingivales contra las bacterias patógenas que ha colonizado la zona supragingival y subgingival. Sin embargo, la evolución y las características clínicas de estas enfermedades están influenciadas por factores adquiridos y genéticos que pueden modificar la susceptibilidad a esta afección como las enfermedades sistémicas. Por lo tanto, las enfermedades periodontales como la periodontitis crónica, periodontitis agresiva y enfermedades ulcero-necrotizantes podrían presentarse como manifestaciones de enfermedades sistémicas ${ }^{4,5}$.

\section{Etiopatogenia de la enfermedad periodontal}

La enfermedad periodontal es el resultado de una compleja interacción entre la biopelícula subgingival y los eventos inflamatorios inmunes en el huésped que se desarrolla en la encía, tejidos gingivales y periodontales, dado como respuesta a la colonización bacteriana.

La entrada de las bacterias y de sus productos favorece el acceso al tejido conectivo provocando una vasodilatación e inflamación de los vasos sanguíneos, aumento en el número de neutrófilos y macrófagos que van a migrar a través del epitelio de unión y llegará a formar una parte principal del infiltrado inflamato- rio, al mismo tiempo que las células más coronales del epitelio de unión comienzan a proliferar con lo que se va a iniciar la formación de bolsa periodontal, aumentado la evolución de la gingivitis a periodontitis ${ }^{6}$.

El síndrome metabólico (SM) es un conjunto de factores de riesgo para desarrollar diabetes mellitus tipo 2 (DM2) y enfermedad cardiovascular (ECV), que se presenta como manifestaciones de un estado de resistencia a la insulina e hiperinsulinismo compensador asociados con trastornos del metabolismo de los carbohidratos y lípidos, cifras elevadas de presión arterial (PA) y obesidad ${ }^{7,8,9}$.

Para diagnosticar este síndrome, un individuo debe presentar tres o más de los siguientes criterios ${ }^{10}$ : resistencia a la insulina, obesidad (aumento del diámetro abdominal), presión arterial elevada, Dislipidemia e hiperglucemia.

Por lo dicho anteriormente, es importante esclarecer la relación entre la enfermedad periodontal y el síndrome metabólico; los resultados del presente estudio permiten evidenciar la estrecha relación que existe entre el SM y la periodontitis, influenciándose una a la otra. Por lo tanto, médicos como odontólogos deben tener en cuenta ambas condiciones para un manejo integral, lograr así una intervención oportuna y evitar la manifestación de la enfermedad periodontal en consecuencia del síndrome metabólico o viceversa, e igualmente que haya diagnóstico temprano con un tratamiento oportuno para disminuir cualquier consecuencia asociada a estas patologías.

\section{MATERIALES Y MÉTODOS}

Se realizó una revisión sistemática, soportada en artículos científicos que evidencian la relación que existe en pacientes con SM y la enfermedad periodontal. Se consultaron 4 bases de datos electrónicas (Dentistry \& Oral Science Source, Pubmed, Web Science y Scopus) empleando términos $\mathrm{MeSH}$, de manera individual y combinada: "Periodontitis"; "Metabolic syndrome"; "Periodontitis AND Metabolic syndrome". Se seleccionaron artículos publicados entre el 2000 y el 2016 en inglés, español y portugués, valorándolos primero por el título, resumen y, finalmente, por el texto completo. 
El instrumento de recolección de datos constó de variables como: objetivo, características de los participantes, género, edad, parámetros, resultados, valores de asociación y conclusiones.

Teniendo en cuenta los parámetros de la Resolución $\mathrm{N}^{\circ} 008430$ del 4 de octubre de 1993, se establece que el estudio es clasificado como "investigación sin riesgo", ya que no se realizó ninguna intervención y la información necesaria se obtuvo y analizó a partir de investigaciones previas ${ }^{11}$.

\section{RESULTADOS}

La estrategia de búsqueda incluyó los siguientes descriptores temáticos, de manera individual y combinada: "Periodontitis"; "Metabolic syndrome"; "Periodontitis AND Metabolic syndrome”. El número de artículos obtenidos en cada una de las bases de datos consultadas se aprecia en el flujograma (Figura 1).

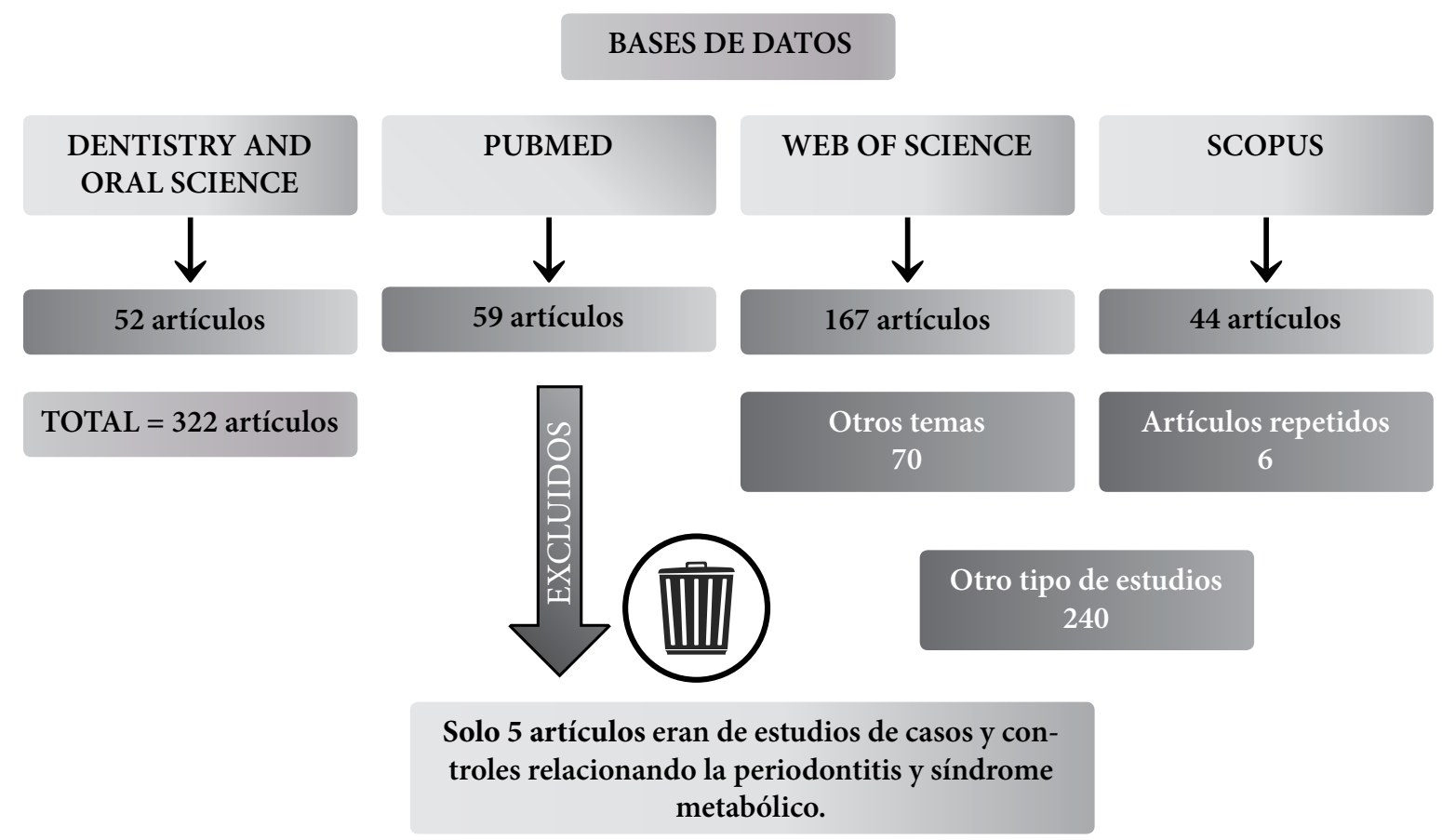

Figura 1. Flujograma de recolección de datos

En total se encontraron 322 artículos en las bases de datos, utilizando los descriptores mencionados, de los cuales, 5 artículos cumplían con los criterios de inclusión con un grado de coincidencia de 66,6\%; 4 artículos fueron encontrados de manera simultánea en las 4 bases de datos Dentistry \& Oral Sciene Source, Pubmed, Web of Science y Scopus. Posteriormente, se realizó la descripción de los artículos incluidos (Tabla 1).

Dos de los artículos estudiaron la influencia del síndrome metabólico sobre la periodontitis valorando variables como profundidad de surco, sangrado al sondaje, índice gingival, reabsorción ósea, índice de placa, pérdida de inserción - nivel de inserción ${ }^{17,18}$. Li y colaboradores, al evaluar 208 participantes encontraron que los pacientes con síndrome metabólico tenían mayores profundidades de surco (OR de 3,2) índices de placa (OR de 47,4 ), mayores pérdidas de niveles de inserción (OR de 15,6$)$ concluyendo así que los pacientes con este síndrome tenían pobres condiciones periodontales independientemente de otros factores de riesgo ${ }^{15}$. Khader y colaboradores, al evaluar 156 participantes, reportaron que el índice gingival $(\mathrm{p}<0,005)$, profundidad de sondaje $(\mathrm{p}<0,005)$ y pérdida del nivel de inserción $(\mathrm{p}<0,005)$ fueron valores significativamente más altos en personas con síndrome metabólico, comparados con las personas sin el síndrome ${ }^{16}$. Todos los artículos tuvieron en cuenta otros factores de riesgo, como fumar, beber alcohol o realización de actividad física, los cuales eran más frecuentes en los $\operatorname{casos}^{12-16}$ (Tabla 3 y Tabla 5). 
Tabla 1. Descripción de artículos incluidos

\begin{tabular}{|c|c|c|c|}
\hline Autores & Revista & País & Objetivo \\
\hline Jaramillo y cols, $2016^{12}$ & Clin Oral Invest & Colombia & $\begin{array}{l}\text { Determinar si existe una relación entre el síndrome metabólico y la } \\
\text { periodontitis crónica en adultos colombianos }{ }^{12} \text {. }\end{array}$ \\
\hline Han y cols., $2012^{13}$ & $\begin{array}{l}\text { Journal of Clinical } \\
\text { Periodontology }\end{array}$ & Korea & $\begin{array}{l}\text { Evaluar si la periodontitis está asociada con el síndrome metabólico } \\
\text { entre koreanos }{ }^{13} \text {. }\end{array}$ \\
\hline Nibali y cols, $2007^{14}$ & $\begin{array}{l}\text { Journal of Clinical } \\
\text { Periodontology }\end{array}$ & Reino Unido & $\begin{array}{l}\text { Investigar la asociación entre la periodontitis severa y el incremento en } \\
\text { inflamación y factores de riesgo metabólico para enfermedad cardio- } \\
\text { vascular }^{14} \text {. }\end{array}$ \\
\hline Li y cols., $2009^{15}$ & $\begin{array}{l}\text { Journal Periodonto- } \\
\operatorname{logy}\end{array}$ & China & Evaluar la asociación de periodontitis con el síndrome metabólico ${ }^{15}$. \\
\hline Khader y cols., $2008^{16}$ & Journal Periodontol & Jordania & $\begin{array}{l}\text { Evaluar la distribución de los parámetros periodontales entre los pa- } \\
\text { cientes con síndrome metabólico y sujetos sin síndrome metabólico en } \\
\text { el norte de Jordán }{ }^{16} \text {. }\end{array}$ \\
\hline
\end{tabular}

Tabla 2. Factores sociodemográficos de los artículos

\begin{tabular}{|c|c|c|c|c|}
\hline \multirow{2}{*}{$\begin{array}{l}\text { Autores } \\
\text { Jaramillo y cols., 2016 }\end{array}$} & \multirow{2}{*}{$\begin{array}{l}\text { Género } \\
\text { : } 416 \\
\text { o: }: 235\end{array}$} & \multirow{2}{*}{$\begin{array}{c}\begin{array}{c}\text { Rango } \\
\text { edad }\end{array} \\
27-84 \text { años }\end{array}$} & \multicolumn{2}{|c|}{$\begin{array}{l}\text { Características de los participantes } \\
\text { casos controles }\end{array}$} \\
\hline & & & CS (431): personas con periodontitis & CT (220): personas sanas (sin periodontitis) \\
\hline Han y cols., $2012^{13}$ & - & No reporta & CS (166): personas con síndrome metabólico & $\begin{array}{l}\text { CT (166): personas sanas (sin síndrome } \\
\text { metabólico) }\end{array}$ \\
\hline Nibali y cols., $2007^{14}$ & $\begin{array}{l}+ \\
+: 264 \\
O: 221\end{array}$ & 38- 48 años & CS (302): personas con periodontitis & CT (183): personas sanas (sin periodontitis) \\
\hline Li y cols., $2009^{15}$ & $\begin{array}{l}\text { o: } 123 \\
\delta:: 85\end{array}$ & 37-68 años & CS (152): personas con síndrome metabólico & $\begin{array}{l}\text { CT (56): personas sanas (sin síndrome } \\
\text { metabólico) }\end{array}$ \\
\hline Khader y cols., $2008^{16}$ & $\begin{array}{l}\text { of: } 100 \\
\lambda^{2}: 56\end{array}$ & 25-76 años & CS (78): personas con síndrome metabólico & $\begin{array}{l}\text { CT ( } 78 \text { ): personas sanas (sin síndrome } \\
\text { metabólico) }\end{array}$ \\
\hline
\end{tabular}

Convenciones. -No reporta, $\uparrow$ (femenino), $\widehat{\partial}$ (masculino).

Tabla 3. Artículos de influencia del síndrome metabólico sobre la periodontitis

\begin{tabular}{lccccc}
\hline \multicolumn{1}{c}{ Referencia } & Profundidad de surco & Índice de placa & Índice gingival & Pérdida de inserción & Conclusión \\
\hline Li y cols., $2009^{15}$ & $\mathrm{OR}=3,2$ & $\mathrm{OR}=47,4$ & - & $\mathrm{OR}=15,6$ & $\mathrm{MS} \approx$ Periodontitis \\
\hline Khader $\mathrm{y}$ cols., $2008^{16}$ & $\mathrm{p}<0,005$ & - & $\mathrm{p}<0,005$ & $\mathrm{p}<0,005$ & $\mathrm{MS} \approx$ Periodontitis \\
\hline
\end{tabular}

Convenciones. - (no reporta), $\approx$ (asociación).

Tres de los artículos estudiaron la influencia de la periodontitis sobre el síndrome metabólico teniendo en cuenta variables, como la presencia de Aggregatibacter actinomycetemcomitans, Porphyromonas gingivalis, Treponema denticola, y Tannerella forsythia, valores de glucosa, índice de masa corporal, presión sanguínea, triglicéridos, lipoproteína de alta y baja densidad (HDL-LDL) y factores inflamatorios ${ }^{12-14}$.
Jaramillo y colaboradores, al evaluar 650 individuos encontraron que las personas con periodontitis tienen un OR de 2,3 para valores de glucosa $\geq 110 \mathrm{mg} /$ $\mathrm{dL}$; OR de 1,4 para valores de ICM $\geq 25 \mathrm{~kg} / \mathrm{m} 2$; OR de 2,13 para presión sanguínea sistólica $\geq 130 \mathrm{mmHg}$; OR de 1,3 para triglicéridos $\geq 150 \mathrm{mg} / \mathrm{dL}$; OR de 0,9 para $\mathrm{HDL} \leq 50 \mathrm{mg} / \mathrm{dL}$, concluyendo que la periodontitis estaba asociada con el síndrome metabólico con un OR 
ajustado de $2,7^{12}$. Han y cols., al evaluar 333 participantes, encontraron que los casos con síndrome metabólico fumaban más (OR de 1,7), tenían más periodontitis (OR de 1,8 ) y presentaban más diabetes mellitus (OR de 4,9), encontrando que la periodontitis puede ser un factor independiente relacionado en el síndrome metabólico ${ }^{13}$. Nibali y cols., al evaluar 485 participantes, encontraron que los pacientes con periodontitis exhibieron mayor número de factores inflamatorios $(\mathrm{p}=0,001)$, tendían a tener mayores niveles de colesterol sérico $(\mathrm{p}<0,001)$, triglicéridos $(\mathrm{p}=0,439)$, glucosa $(\mathrm{p}=0,003)$; sugiriendo un posible enlace entre la periodontitis y el síndrome metabólico ${ }^{14}$ (Tabla 4 y Tabla 5).

Tabla 4. Artículos de influencia de la periodontitis sobre el síndrome metabólico

\begin{tabular}{|c|c|c|c|c|c|}
\hline Referencia & Glucosa & Índice de masa corporal & Presión sanguínea & Triglicéridos/colesterol & Conclusión \\
\hline Jaramillo y cols., $2016^{12}$ & $\mathrm{OR}=2,3$ & $\mathrm{OR}=1,4$ & $\mathrm{OR}=2,1$ & $\mathrm{OR}=1,3$ & $\begin{array}{l}\text { Periodontitis } \approx \mathrm{MS} \\
\mathrm{OR}=2,7\end{array}$ \\
\hline Han y cols., $2012^{13}$ & $\mathrm{OR}=4,9$ & - & - & - & $\begin{array}{l}\text { Periodontitis } \approx \mathrm{MS} \\
\mathrm{OR}=1,8\end{array}$ \\
\hline Nibali y cols., $2007^{14}$ & $\mathrm{p}=0,003$ & - & - & $\mathrm{p}=0,4 / \mathrm{p}<0,001$ & $\begin{array}{l}\text { Periodontitis } \approx \text { inflamación } \\
\text { sistémica } \approx \mathrm{MS}\end{array}$ \\
\hline
\end{tabular}

Convenciones. - (no reporta), $\approx$ (asociación).

Tabla 5. Resultados y conclusiones de los estudios

\begin{tabular}{|c|c|c|}
\hline Referencia & Parámetros & Resultados \\
\hline Jaramillo y cols., $2016^{12}$ & PCR, Glucosa, ICM, PA, TGD HDL, Fumar & $\begin{array}{l}\text { Pts con periodontitis: } \uparrow \text { presencia de bacterias según PCR, } \uparrow \text { Gluco- } \\
\text { sa, } \uparrow \text { ICM, } \uparrow P A, \uparrow \text { TGD, } \downarrow \text { HDL, } \uparrow \text { Fumar }\end{array}$ \\
\hline Han y cols., $20121^{3}$ & IP, Fumar, beber alcohol, actividad física & Pts con MS: $\uparrow$ Fumar, $\uparrow$ beber alcohol, $\downarrow$ actividad física \\
\hline Nibali y cols., $2007^{14}$ & WBC, IF, LDL, HDL, TGD, Glucosa, Fumar & $\begin{array}{l}\text { Pts con periodontitis: } \uparrow \mathrm{WBC}, \uparrow \mathrm{IF}, \uparrow \mathrm{LDL}, \downarrow \text { HDL, } \uparrow \mathrm{TGD}, \uparrow \text { Gluco- } \\
\text { sa, Fumar }\end{array}$ \\
\hline Li y cols., $2009^{15}$ & AL, PD, BOP, IP, Fumar & Pts con MS: $\uparrow A L, \uparrow P D, \uparrow B O P, \uparrow P I, \uparrow F u m a r$ \\
\hline Khader y cols., $2008^{16}$ & CAL, IP, IG, PD, Fumar & Pts con MS: $\uparrow \mathrm{CAL}, \uparrow \mathrm{IP}, \uparrow \mathrm{IG}, \uparrow \mathrm{PD}, \uparrow$ Fumar \\
\hline
\end{tabular}

Convenciones. ICM (índice de masa corporal), PA (presión arterial), TGD (triglicéridos), HDL (lipoproteína de alta densidad), PD (profundidad de surco), BOP (sangrado al sondaje), IG (índice gingival), RO (reabsorción ósea), WBC (número ajustado de leucocitos), IF (factores inflamatorios), LDL (lipoproteína de baja densidad), AL (pérdida de inserción), IP (índice de placa), CAL (nivel de inserción clínica), $\uparrow($ incremento), $\downarrow$ (decrecimiento).

\section{DISCUSIÓN}

El presente estudio se realizó con el objetivo de determinar la relación del síndrome metabólico con la periodontitis a través de una revisión sistemática, dada la naturaleza inflamatoria de las dos entidades y análisis de estudios de casos y controles. El SM es un conjunto de factores de riesgo de enfermedad cardiovascular que incluye obesidad, hiperglicemia, hiperlipidemia y presión arterial ${ }^{10}$. La enfermedad periodontal se caracteriza por la pérdida de inserción gingival, formación de bolsas periodontales y la resorción ósea que resultan de la infección por patógenos periodontales. Se encontró que los valores de los índices periodontales y el estado de los tejidos de soporte dentario empeoraban con la presencia del síndrome metabólico. De igual manera, se encontró que los parámetros del síndrome metabólico eran mayores cuando los pacientes presentaban periodontitis.

Según Kumar y colaboradores, en un estudio de corte transversal encontraron una fuerte asociación entre la periodontitis crónica y el SM con un OR de 2,6. Teniendo en cuenta variables como niveles de triglicéridos, presión sanguínea y niveles de azúcar. La asociación encontrada fue independientemente de otros factores de riesgo, como la edad, género o consumo de tabaco ${ }^{17}$. Para Gomes y colaboradores, mediante otro estudio de corte transversal se valoró la influencia de la periodontitis sobre el síndrome meta- 
bólico, encontrando una asociación $(\mathrm{OR}=2,1)$ al tener en cuenta otros factores de riesgo y la prevalencia del SM estuvo relacionada con la periodontitis severa ${ }^{18}$.

Thanakun y colaboradores, en su estudio encontraron que todas las condiciones periodontales eran peores en pacientes con síndrome metabólico con un OR de 3,6 y entre más componentes del síndrome metabólico estaban presentes había una mayor asociación con la periodontitis, los componentes por separado no tuvieron asociación excepto la presión arterial ${ }^{19}$.

Watanabe y colaboradores, mediante una revisión de publicaciones, reportaron que todos los estudios demostraban una relación positiva entre la periodontitis y el síndrome metabólico, pero fue difícil determinar la contribución de la periodontitis al SM, esto se debe a la diferencia de criterios en el diagnóstico periodontal ${ }^{20}$. Lo cual también sucedió en el presente estudio, debido a los diferentes criterios que cada artículo tuvo en cuenta fue difícil encaminar el análisis de los resultados hacia una misma dirección.

Bullon y colaboradores, en su revisión llegaron a la conclusión de que el estrés oxidativo de las células puede actuar como un enlace común en la relación entre el SM y la periodontitis, porque debido a este se influencian el uno al otro bidireccionalmente ${ }^{21}$. Gurav, mediante una revisión, encontró que los estudios actuales han mostrado una relación coherente entre la periodontitis y el síndrome metabólico, cuando se realiza la terapia periodontal individual se reduce la incidencia de varias complicaciones sistémicas ${ }^{22}$.

Los resultados del presente estudio permiten evidenciar la estrecha relación que existe entre el síndrome metabólico y la periodontitis, influenciándose una a la otra. Por lo tanto, en la práctica odontológica como en la médica se debe observar al paciente de una manera completa e integral, y así lograr una intervención oportuna, y evitar la manifestación de la enfermedad periodontal y en consecuencia del síndrome metabólico o viceversa, obteniendo un diagnóstico temprano con un tratamiento oportuno para disminuir cualquier consecuencia asociada a estas patologías.

Entre las limitaciones de este estudio se encuentra el tamaño de muestra, ya que en total se analizaron cinco artículos. Además, fue complejo reportar las características comunes entre los artículos que eran considerablemente diferentes. Sin embargo, los objetivos fueron cumplidos y reportados de la mejor manera.

\section{CONCLUSIÓN}

De acuerdo con los estudios analizados sobre la relación del síndrome metabólico con la periodontitis, se observó que existe una relación estrecha entre ellos al obtener valores de odd ratio (OR) mayores a 1 en las variables influyentes, como índice de masa corporal, glucosa, lípidos, presión arterial, profundidad de surco, sangrado al sondaje, índice gingival, reabsorción ósea y nivel de inserción.

Con la presencia del síndrome metabólico, la salud de los tejidos periodontales disminuye, empeorándose aún más cuando los niveles de parámetros sistémicos aumentan. Condiciones periodontales como índices de sangrado, pérdida ósea, niveles de inserción y profundidades de surco indican una periodontitis con mayor severidad que en los pacientes sin el síndrome.

Se establece que el síndrome metabólico y la periodontitis tienen una relación recíproca, porque cuando los estudios valoraban la influencia del SM sobre la periodontitis o la influencia de la periodontitis sobre el SM, en ambas situaciones encontraban asociación.

\section{BIBLIOGRAFÍA}

1. Alonso Rosado A, Hernández Marcos G, Pérez Gómez R. Evidencias científicas de la relación entre periodontitis y enfermedades cardiovasculares. Avances en Periodoncia e Implantología Oral. 2008;20(3):173-181.

2. Colombia. Ministerio de Salud. Centro Nacional de Consultoría. Estudio Nacional de Salud Bucal 2013-2014, En: IV Estudio Nacional de Salud Bucal. Bogotá, 2014.

3. Peña Sisto M, Peña Sisto L, Díaz Felizola Á, Torres Keiruz D, Lao Salas N. La enfermedad periodontal como riesgo de enfermedades sistémicas. Revista Cubana de Estomatología. 2008;45(1):1-9.

4. Andriankaja O, Sreenivasa S, Dunford R, DeNardin E. Association between metabolic syndrome and periodontal disease. Australian Dental Journal. 2010;55(3):252259. doi:10.1111/j.1834-7819.2010.01231.x. 
5. Sanz-Sánchez I, Bascones-Martínez A. Otras enfermedades periodontales: I: Periodontitis como manifestación de enfermedades sistémicas. Avances en Periodoncia [Internet] [Fecha de acceso: 10 de abril de 2015]. 2008;20(1):59-66.

6. Newman, MG, Takei H, H. Carranza. Periodontología clínica. 2004:65-75.

7. Grundy SM, Brewer HB Jr, Cleeman JI, Smith SC Jr, Lenfant C. Definition of metabolic syndrome: report of the National Heart, Lung, and Blood Institute/American Heart Association conference on scientific issues related to definition. Arterioscler Thromb Vasc Biol. 2004;24:e1318. doi:10.1161/01.ATV.0000111245.75752.C6.

8. López ME, Sosa MA, Labrousse NPM. Síndrome metabólico. Revista de Posgrado de la VIa Cátedra de Medicina. 2007;174:12-15.

9. Benguigui C, Bongard V, Ruidavets J, Chamontin B, Sixou $\mathrm{M}$, Amar J et al. Metabolic syndrome, insulin resistance, and periodontitis: a cross-sectional study in a middle-aged French population. Journal of Clinical Periodontology. 2010;37(7):601-608. Available from: Dentistry \& Oral Sciences Source. doi:10.1111/j.1600-051X.2010.01571.x.

10. Alberti KGMM, Eckel RH, Grundy SM, Zimmet PZ, Cleeman JI, Donato K et al. Harmonizing the metabolic syndrome: a joint interim statement of the International Diabetes Federation Task Force on Epidemiology and Prevention; National Heart, Lung, and Blood Institute; American Heart Association; World Heart Federation; International Atherosclerosis Society; and International Association for the Study of Obesity. 2009;120(11):16405. doi: 10.1161/CIRCULATIONAHA.109.192644.

11. Ley 23 de 1982. Dada en Santafé de Bogotá D.C.

12. Jaramillo A, Contreras A, Lafaurie GI, Duque A, Ardila $\mathrm{CM}$, Duarte $\mathrm{S}$ et al. Association of metabolic syndrome and chronic periodontitis in Colombians. Clin Oral Investig. Aug 17. 2016. doi: 10.1007/s00784-016-1942-9.

13. Han D-H, Lim S, Paek D, Kim H-D. Periodontitis could be related factors on metabolic syndrome among Koreans: a case-control study. J Clin Periodontol. 2012;39:30-37. doi: 10.1111/j.1600-051X.2011.01806.x.
14. Nibali L, D’Aiuto F, Griffiths G, Patel K, Suvan J, Tonetti MS. Severe periodontitis is associated with systemic inflammation and a dysmetabolic status: a case-control study. Journal of Clinical Periodontology. 2007;34(11):931-937. doi: 10.1111/j.1600-051X.2007.01133.x.

15. Li P, He L, Sha Y, Luan Q. Relationship of metabolic syndrome to chronic periodontitis. J Periodontol. 2009;80(4):541-549. doi: 10.1089/met.2016.0018.

16. Khader Y, Khassawneh B, Obeidat B, Hammad M, El-Salem K, Bawadi $\mathrm{H}$ et al. Periodontal status of patients with metabolic syndrome compared to those without metabolic syndrome. J Periodontol. 2008;79(11):2048-2053. doi: 10.1902/jop.2008.080022.

17. Kumar N, Bhardwaj A, Negi PC, Jhingta PK, Sharma D, Bhardwaj VK. Association of chronic periodontitis with metabolic syndrome: A cross-sectional study. Journal of Indian Society of Periodontology. 2016;20(3):324-329.

18. Gomes-Filho I, Conceição Mercês M, de Santana PassosSoares J, Seixas da Cruz S, Teixeira Ladeia AM, et al. Severity of Periodontitis and Metabolic Syndrome: Is There an Association. Journal of Periodontology. 2015;87(4):357366. doi: 10.1902/jop.2015.150367.

19. Thanakun S, Watanabe H, Thaweboon S, Izumi Y. Association of untreated metabolic syndrome with moderate to severe periodontitis in Thai population. Journal of periodontology. 2014;85(11):1502-1514. 0.1902/ jop.2014.140105.

20. Watanabe K, Cho Y. Periodontal Disease and Metabolic Syndrome: A Qualitative Critical Review of their Association. Archives of Oral Biology. 2014;59(8):855-870. doi: 10.1016/j.archoralbio.2014.05.003.

21. Bullon P, Morillo JM, Ramirez MC, Quiles JL, Newman HN, Battino M. Metabolic Syndrome and Periodontitis: Is Oxidative Stress a Common Link. J Dent Res. 2009;88(6):503-518. doi: 10.1177/0022034509337479.

22. Gurav AN. The association of periodontitis and metabolic syndrome. Dental Research Journal. 2014;11(1):1-10. 